\title{
L'HOMME L'Homme
}

Revue française d'anthropologie

164 | octobre-décembre 2002

Histoire, littérature et ethnologie

\section{Thierry Wendling, Ethnologie des joueurs d'échecs}

Publié avec le concours du Fonds national suisse de la recherche scientifique. Paris, PUF, 2002, 256 p., bibl., index, ph., ill.

(«Ethnologies »)

\section{Gaetano Ciarcia}

\section{OpenEdition}

Journals

Édition électronique

URL : https://journals.openedition.org//homme/14232

DOI : 10.4000//homme.14232

ISSN : 1953-8103

Éditeur

Éditions de l'EHESS

Édition imprimée

Date de publication : 1 décembre 2002

Pagination : 187-188

ISBN : 2-7132-1775-X

ISSN : 0439-4216

\section{Référence électronique}

Gaetano Ciarcia, «Thierry Wendling, Ethnologie des joueurs d'échecs », L'Homme [En ligne], 164 octobre-décembre 2002, mis en ligne le 25 mars 2008, consulté le 24 avril 2022. URL : http:// journals.openedition.org//homme/14232 ; DOI : https://doi.org/10.4000//homme.14232

Ce document a été généré automatiquement le 24 avril 2022

(c) École des hautes études en sciences sociales 


\section{Thierry Wendling, Ethnologie des joueurs d'échecs}

Publié avec le concours du Fonds national suisse de la recherche scientifique. Paris, PUF, 2002, 256 p., bibl., index, ph., ill.

(« Ethnologies »)

\section{Gaetano Ciarcia}

1 CE LIVRE nous invite à un voyage ethnologique dans «Le monde des échecs ", pour reprendre le titre de la première partie. Avant de présenter et d'interpréter l'histoire de l'organisation sociale de ce "monde », Thierry Wendling pose, dans deux chapitres introductifs, les jalons de son étude en déblayant le terrain de certaines façons d'appréhender le jeu qui, à son avis, en altèrent la compréhension. Ainsi, délaissant la figure idéale du joueur, il s'appuie sur l'observation des joueurs, de leurs pratiques et de leurs espaces de sociabilité; il prend ses distances vis-à-vis de toute approche empathique ou "mystique» (p.33), de la tentation de capter une prétendue essence culturelle du jeu et de l'idée du jeu comme "reflet» (p. 40), archétype, inversion ou projection ludiques de l'ordre sociétal. Wendling s'attache à saisir le «statut théorique du joueur ordinaire » (p. 10) en relation avec le dynamisme de la pratique échiquéenne. Il s'intéresse aux notions de règle et de sociabilité qu'il analyse par rapport à cette "nécessité de la croyance » (p. 11) liée à la fabrication des significations culturelles et sociales dans le jeu. Lui-même joueur d'échecs classé international, l'auteur associe une connaissance approfondie du milieu, due à sa situation particulière d'informateur privilégié de son propre travail, à une distanciation critique inhérente à sa démarche heuristique. Par ailleurs, son écriture limpide permet à ceux qui ignorent les règles du jeu d'interroger les représentations que s'en font les joueurs, mais aussi aux joueurs occasionnels, comme l'auteur de ce compte rendu, de mieux concevoir le continuum et les ruptures contextuelles entre les échecs comme activité de loisir et le champ sociologique déterminé par les organes officiels, nationaux et internationaux.

Dans la première partie, Wendling décrit une communauté, celle des joueurs membres de la Fédération française des échecs, à travers les «savoirs, techniques, croyances, histoires et anecdotes, sentiments esthétiques, formes de sociabilité, coutumes, etc., 
partagés par les joueurs de compétition [qui] forment un ensemble cohérent, autrement dit une manière particulière de penser le monde, de définir les relations entre les hommes, et conjointement d'agir et d'interagir dans ce monde » (p. 49). En ce sens, les équipes, les clubs et les tournois sont pour l'auteur autant de lieux qui alimentent sa réflexion sur la culture échiquéenne, qu'il considère aussi sous l'angle du devenir historique de ses formes d'institutionnalisation. Au fil de cette restitution critique, nous sommes confrontés à la stratification d'une tradition et de ses avatars discursifs, sans jamais perdre de vue sa dimension "vivante", à savoir l'entrecroisement des différents niveaux d'interaction, d'interconnaissance et de hiérarchisation qui la composent.

Dans la seconde partie, "Variantes échiquéennes », l'auteur examine la pensée propre au jeu d'échecs. Il voit dans la dimension orale d'une pratique fondée sur une production écrite imposante, à la fois savante et vulgarisatrice - relative à la transmission de ses règles et à la transcription tant algébrique que descriptive des parties -, un antagonisme d'ordre cognitif qui, à l'intérieur du jeu, structurerait la relation entre «mémoire humaine et mémoire écrite» (p. 184). Si, «le temps de la partie, la pensée échiquéenne veut se croire affranchie de ce qui lui donne sa substance " (ibid.), le dualisme entre l'écrit et l'oral n'est pas le seul à participer aux explications que les joueurs se donnent à eux-mêmes et qui sembleraient lier et opposer «la dialectique des stratégies à la mécanique du modèle »1 . Le temps, ou plutôt les temps du jeu, réaliseraient, en l'accomplissant spatialement, toute la complexité due à la discordance intrinsèque à la durée, double en quelque sorte, de la partie. Car c'est l'action concomitante de deux dynamiques conflictuelles - la succession des coups et l'avancée irréversible de la pendule comptant le temps imparti à chacun des deux adversaires - qui constitue la mesure, temporelle bien sûr, mais aussi ontologique ou, si l'on veut, fondatrice, de l'affrontement et lui donne un sens décisif intégrant celui, primordial, de la victoire ou de la défaite. Wendling relie ce caractère binaire à la description qu'en font les joueurs aux prises, durant la partie, à un espace, l'échiquier, où la position toujours passagère (jusqu'à la fin de la rencontre) des pièces et de leurs pousseurs constitue la scène mouvante, avec ses marques écrites (les annotations au moyen desquelles les joueurs doivent transcrire le cours de la partie), d'un « immense et compliqué palimpseste de la mémoire où l'on ne peut que ressentir l'indistinct passage du temps » (p. 225).

4 Thierry Wendling précise en conclusion qu'il ne considère pas son ethnographie comme achevée et qu'il entend la poursuivre en comparant la pratique et la pensée des échecs dans différents pays. Son mérite, qu'il faut saluer, reste d'avoir ouvert un nouveau champ d'investigation à la discipline anthropologique.

\section{NOTES}

1. Pierre Bourdieu, Le Sens pratique, Paris, Éditions de Minuit, 1980 : 170. 


\section{AUTEUR}

GAETANO CIARCIA

Université Montpellier-III, Centre d'études et de recherches comparatives en ethnologie, Montpellier. 